\title{
Effect of Physical Activity on the Association Between Dietary Fiber and Constipation: Evidence From the National Health and Nutrition Examination Survey 2005-2010
}

\author{
Yi Li, ${ }^{1}$ Wei-Dong Tong, ${ }^{2 *}$ and Yang Qian ${ }^{3}$ \\ ${ }^{I}$ Medical College of Yan'an University, Yan'an, Shaanxi, China; ${ }^{2}$ Gastric and Colorectal Division, Department of General Surgery, Daping
} Hospital, Army Medical University, Chongqing, China; and ${ }^{3}$ Operating Room, Shaanxi Provincial People's Hospital, Xi'an, Shaanxi, China

\section{Background/Aims}

The effect of physical activity on the relationship between dietary fiber intake and constipation has not been comprehensively studied. This study aims to explore the impact of physical activity.

\section{Methods}

Data were obtained from 3 cycles of the National Health and Nutrition Examination Survey (NHANES) 2005-2010 and included a total of 13941 participants aged $\geq 20$ years. Multiple logistic regression analysis was used to investigate the independent association between dietary fiber and constipation. Interaction analysis was also performed to analyze the relationship between dietary fiber and constipation in different physical activity groups.

\section{Results}

Among non-active participants, dietary fiber intake did not associate with stool consistency $(\mathrm{OR}, 1.02 ; 95 \% \mathrm{Cl}, 0.98-1.05 ; P=$ 0.407). For physically active participants, 1-gram unit increase in dietary fiber intake reduced the risk of stool consistency by $3 \%$ (OR, $0.97 ; 95 \% \mathrm{Cl}, 0.94-0.99 ; P=0.020)$. Moreover, the relationship between dietary fiber intake and stool consistency was significantly different for groups with different levels of physical activity $(P$ interaction $=0.044)$. However, dietary fiber intake was not related to stool frequency among non-active participants $(\mathrm{OR}, 0.99 ; 95 \% \mathrm{Cl}, 0.94-1.05 ; P=0.767)$ nor physically active participants $(\mathrm{OR}, 1.01$; $95 \% \mathrm{Cl}, 0.97-1.04 ; P=0.751)$.

\section{Conclusions}

Increasing dietary fiber intake was associated with stool consistency-related constipation among physically active participants, but not among non-active participants. However, increasing dietary fiber intake is not significantly associated with stool frequency in different physical activity groups.

(J Neurogastroenterol Motil 2021;27:97-109)

\section{Key Words}

Constipation; Dietary fiber; Exercise; Nutrition surveys 
Received: March 16, 2020 Revised: May 27, 2020 Accepted: July 21, 2020

(a) This is an Open Access article distributed under the terms of the Creative Commons Attribution Non-Commercial License (http://creativecommons. org/licenses/by-nc/4.0) which permits unrestricted non-commercial use, distribution, and reproduction in any medium, provided the original work is properly cited.

*Correspondence: Wei-Dong Tong, MD, PhD

Gastric and Colorectal Division, Department of General Surgery, Daping Hospital, Army Medical University, 10 Changjiang Zhi Lu. Chongqing 400042, China

Tel: +86-23-68729356, E-mail: vdtong@163.com; tongweidong@gmail.com

\section{Introduction}

Constipation is characterized by lumpy or hard stools, decreased stool frequency, feelings of incomplete evacuation, etc. ${ }^{1}$ The stool consistency and stool frequency are important symptoms in the diagnosis of constipation. ${ }^{1,2}$ The overall prevalence of constipation is reported to range from $7 \%$ to $10 \%$ in adults. ${ }^{3}$ The prevalence of constipation is different between men and women, ${ }^{4}$ which is $6 \%$ higher in women than that of men. ${ }^{5}$ Furthermore, the incidence of constipation varies by age, with a rate of $33 \%$ in people over 60 years of age ${ }^{6}$ and tend to rise in people with lower socio-economic status. $^{7}$

Constipation is associated with poor quality of life, ${ }^{8,9}$ including anxiety, depression, obsessive-compulsive traits, and somatization. ${ }^{10}$ Besides, constipation increases healthcare costs. It has been estimated that the annual cost of over-the-counter laxatives to alleviate constipation is $\$ 821$ million in America. ${ }^{11}$ Many patients have sought medical help to relieve constipation, however, about half of them are dissatisfied. ${ }^{8}$ Generally, they are recommended to eat more fiber and have more physical exercise. Given that constipation is a heterogeneous, polysymptomatic, multifactorial disease, ${ }^{12}$ the outcome of management for constipation including dietary modification, medication, and physical therapy are always disappointing. ${ }^{13}$ The relationship between dietary modification and constipation has been studied with varying results. Shen et a ${ }^{14}$ conducted a cross-sectional study and suggested that the low dietary fiber intake increased the risk of constipation. On the contrary, Markland et al, ${ }^{5}$ based on NHANES data analysis, reported that constipation was not related to dietary fiber intake. Thus, whether increasing dietary fiber intake correlates with constipation is controversial.

On the other hand, the impact of physical activity on constipation also attracted much attention in recent years. Applying the NHANES database, Wilson ${ }^{15}$ reported that physical inactivity is not significantly related to stool consistency or stool frequency. In contrast, Dukas et $\mathrm{al}^{16}$ conducted a study of nurses' health and concluded that women who increase dietary fiber intake and perform moderate physical activity are less likely to develop constipation.
To date, no publications have been retrieved considering the relationship between dietary fiber intake and constipation at different levels of physical activity. Therefore, we aim to address the novel question of whether there is a relationship between dietary fiber intake and constipation at different levels of physical activity. The NHANES database is a population-representative survey that provides nationwide estimates of various health parameters, which is helpful for us to address the problem. Similar to the previous studies, ${ }^{14,15}$ we designed a cross-sectional study utilizing the NHANES database.

\section{Materials and Methods}

\section{Data Source}

The NHANES is a survey research program consisting of cross-sectional surveys that use a complex, stratified, multistage probability cluster design to collect and analyze data that are representative of the national, non-institutionalized population in America. The National Center for Health Statistics conducts the program and its institutional review board approved this protocol. Since the NHANES data are de-identified, data analysis does not need institutional review board approval or written informed consent by the study subjects. The survey data are freely downloaded from the Centers for Disease Control and Prevention. The data consist of demographic characteristics, physical examination results, laboratory results, and questionnaire survey items.

The 2005-2010 NHANES dataset is comprised of 17132 participants aged $\geq 20$ years old. We eliminated participants who lacked data on stool consistency and frequency, including 6 participants who refused, 161 participants who did not know, and 2374 participants who were missing. Then, there were 14591 eligible participants. Next, 243 participants were excluded for missing dietary fiber intake data and 407 pregnant women were excluded. The final cohort to be analyzed included 13941 participants (Fig. 1).

\section{Definition of Constipation}

Based on the NHANES database, the previous studies used 


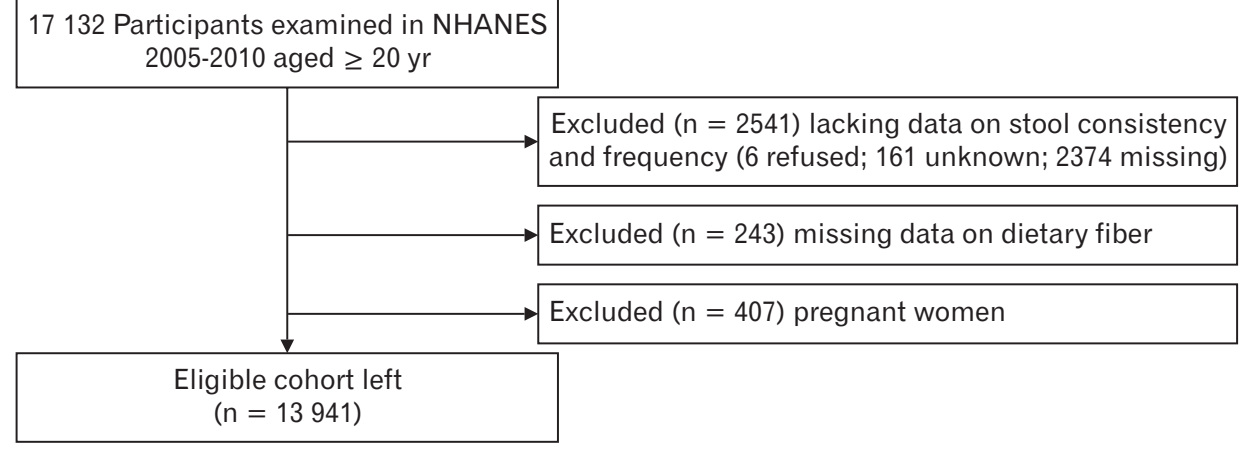

Figure 1. A flow chart of the process for the selection of eligible participants using data from the National Health and Nutrition Examination Survey (NHANES) 2005-2010. stool consistency or stool frequency to define constipation. ${ }^{5,15}$ One of the previous studies reported that stool consistency has little to do with stool frequency, ${ }^{5}$ therefore, we used either stool consistency or stool frequency to define constipation in this study. Before data collection, stool consistency and stool frequency were recorded for 30 days. The Bristol stool form scale, ${ }^{17}$ which includes a variety of color picture cards and detailed descriptions of the 7 stool types, was used to estimate stool consistency. Based on previous research, ${ }^{18}$ constipation was defined as Type 1 (separate hard lumps, like nuts) or Type 2 (sausage-like, but lumpy). The Bristol stool form scale types 3-7 were defined as non-constipation. ${ }^{14}$

Stool frequency was assessed with the following question: "How many times a week do you usually have a bowel movement?" Participants' response levels ranged from 1 to 70 bowel movements (BMs) per week. The results of stool frequency were classified as $<3$ BMs per week (constipation) or $\geq 3$ BMs per week (nonconstipation).

To avoid bias caused by the different definitions of constipation, we carried out a sensitivity analysis focused on other 3 constipationrelated symptoms which were selected from the bowel health questionnaire (BHQ). The first is about self-reported constipation in the past 12 months (BHQ080). If the participants answered "never" to the question: "During the past 12 months, how often have you been constipated," they were classified as self-reported with no constipation. The second is about the laxatives use status (BHQ100). The laxative use status was divided into 2 categories: unused and used. The last is about the frequency of laxative use in the past 30 days (BHQ110). According to the participants' answers to the question, the frequency of laxative use was classified as infrequent use (once a month, 2-3 times a month, and 1-3 times a month) and frequent use (most days). Since only the BHQ from 2009-2010 contained these 3 symptoms, we conducted the sensitivity analysis on the data from 2009-2010.

\section{Dietary Measures}

Based on face-to-face interviews between trained interviewers and respondents, data on the participants' dietary intake were obtained. Information on all foods and beverages consumed 24 hours before the interview (midnight to midnight) was collected. Some variables were related to constipation in previous studies. ${ }^{5,14}$ The dietary variables evaluated in this study were the dietary factors from the NHANES database: dietary fiber, total fat, carbohydrates, protein, cholesterol, total saturated fatty acids, moisture, alcohol, and milk.

The following dietary factors were divided into tertiles of baseline concentrations: dietary fiber $(\mathrm{T} 1<10.7$; $\mathrm{T} 2,10.7-17.9$; $\mathrm{T} 3$ $\geq 18.0 \mathrm{~g} /$ day); total fat $(\mathrm{T} 1<50.6 ; \mathrm{T} 2,50.6-82.2 ; \mathrm{T} 3 \geq 82.3 \mathrm{~g} /$ day); carbohydrate (T1 < 186.0; T2, 186.0-278.3; T3 $\geq 278.4 \mathrm{~g} /$ day); protein ( $\mathrm{T} 1<52.3$; T2, 52.3-81.4; T3 $\geq 81.5 \mathrm{~g} /$ day); cholesterol (T1 < 133.0; T2, 134.0-270.0; T2 $\geq 271.0 \mathrm{mg} /$ day); and total saturated fatty acids $(\mathrm{T} 1<16.6 ; \mathrm{T} 2,16.7-27.7 ; \mathrm{T} 3 \geq 27.8 \mathrm{~g} /$ day).

Moisture was included in the study because it was previously found to be related to constipation. ${ }^{5}$ Moisture was defined as moisture contained in foods and beverages and was also divided into tertiles of baseline concentrations $(\mathrm{T} 1<1572.0$; $\mathrm{T} 2,1572.0-2587.8$; T3 $\geq 2587.9 \mathrm{~g} /$ day). We included alcohol intake because previous studies have suggested that it is related to gastrointestinal function. ${ }^{19}$ Alcohol was divided into 2 categories according to the median $(<6$, $\geq 6 \mathrm{~g} /$ day). Milk was included because it was considered a covariate in a previous study ${ }^{20}$ and was divided into 4 categories: never consume, rarely consume (less than once a week), occasionally consume (once a week or more, but less than once a day), and often consume (once a day or more).

\section{Demographics}

Demographic data was collected through face-to-face visits to 
the participants' households. Information on age, gender (male and female), ethnicity (non-Hispanic white, non-Hispanic black, Mexican American, and other), education (less than 12th grade, high school, and college), marital status (never married, married or living with a partner, and widowed/divorced/separated), and incometo-poverty ratio $(<2, \geq 2)$ were obtained from demographic questionnaires. We considered the income-to-poverty ratio as a confounding variable in this study because it was previously found to be associated with constipation. ${ }^{21}$

\section{Physical Activity}

Information about physical activity was collected during the household interviews utilizing the Global Physical Activity Questionnaire. Participants reported their weekly time spent exercising, which was multiplied by the metabolic equivalent of task (MET) for that activity as defined before 2007. Then, we added the METminutes per week for all activities to get the total MET-minutes per week. Because the NHANES physical activity questionnaire was changed after 2007, we chose moderate work activity, vigorous work activity, moderate recreational activity, vigorous recreational activity, and walking and biking for transportation to calculate the MET-minutes per week. For a particular type of physical activity reported by a participant, he or she reported the amount of time (in minutes) spent on that type of activity on a typical day. Then, the number of minutes per week for each activity was calculated by multiplying the reported number of days by the typical amount of time per day for that activity. The MET-minutes per week was calculated by multiplying the standard MET value of each activity by the total number of minutes per week of each activity. Finally, the total MET-minutes per week of all activities was calculated by summing the MET-minutes per week of each activity. This physical activity quantification method was identical to that in previous studies. ${ }^{14,15}$ According to the United States Department of Health and Human Services, ${ }^{22}$ participants with a MET-minutes per week of $<500$ were defined as non-active, whereas those with $\geq 500$ were considered physically active.

\section{Comorbidities}

According to a previous study, ${ }^{5}$ the participants were grouped based on their number of chronic diseases (none, 1, 2, or more) including arthritis, chronic lung disease (emphysema, chronic bronchitis, and asthma), chronic heart disease (congestive heart failure, coronary heart disease, angina, and heart attack), stroke, any liver condition, and cancer. Asthma was self-reported by participants and defined as having been told by a doctor or health professional that they have asthma and still have asthma now. Chronic bronchitis was identified for those who responded "yes" to the question: "Has a doctor or health professional ever told you that you have chronic bronchitis?" and "Do you still have chronic bronchitis?" Diabetes was identified for those who responded "yes" to the question: "Has a doctor told you that you have diabetes?" or "Are you taking insulin now?" Other diseases were defined based on whether participants self-reported themselves with diseases.

It has been shown that depression is related to constipation. ${ }^{23}$ According to a prior report, ${ }^{24}$ a Patient Health Questionnaire (PHQ-9) score $\geq 10$ is defined as depression.

The oral health condition was also included in the current study based on a previous study that reported its relationship with constipation. ${ }^{25}$ Each participant's dental condition was obtained from the oral health questionnaire that was completed before the physical examination by the computer-assisted personal interview system (interviewer-administered). Poor oral health was defined as those who responded "poor" to the question: "How would you describe the condition of your teeth?"

\section{Other Variables}

Body mass index (BMI) was obtained from physical examination data that were collected by trained health technicians. Based on the criteria of the World Health Organization, BMI was divided into 3 categories: normal, overweight, or obese $(<25,25-29.9, \geq$ $30 \mathrm{~kg} / \mathrm{m}^{2}$, respectively).

It has been reported that smoking was associated with constipation, ${ }^{26}$ we included this in our data analysis. Trained interviewers collected smoking-related data from one-on-one interviews in accordance with the NHANES Mobile Examination Center InPerson Dietary Interviewers Procedure Manual. ${ }^{27}$ According to the answer to the question "Do you smoke now?" smoking status was classified as non-smoking or current smoking.

A previous study suggested that levels of serum 25-hydroxyvitamin $\mathrm{D}$ were related to constipation, ${ }^{28}$ so we incorporated this into this study. Participants' serum levels of 25-hydroxyvitamin D were measured at laboratories of the National Center for Environmental Health at the Centers for Disease Control and Prevention. ${ }^{29}$ Serum 25 -hydroxyvitamin D levels $\leq 50 \mathrm{nmol} / \mathrm{L}(20 \mathrm{ng} / \mathrm{mL})$ were classified as vitamin $\mathrm{D}$ deficiency. ${ }^{30}$

\section{Statistical Methods}

According to the NHANES analytical guidelines, ${ }^{31}$ sample weights from the dietary interviews were re-weighted to merge 6 years' worth of survey data from NHANES 2005 to 2010 
which address nonresponse, noncoverage, and unequal probabilities of selection; this was consistent with the weight method of a prior study. ${ }^{14,15}$ New weights were calculated as $\mathrm{WT}_{05-10}=$ $(1 / 3) \times \mathrm{WTDRD}_{05-06}+(1 / 3) \times \mathrm{WTDRD1}_{07-08}+(1 / 3) \times$ WTDRD1 $1_{09-10}$, where WTDRD1 $1_{05-06}, \mathrm{WTDRD1}_{07-08}$, and WTDRD $1_{09-10}$ are the variable WTDRD1 from the NHANES 20052006, NHANES 2007-2008, and NHANES 2009-2010 dietary interviews, respectively. Furthermore, in line with the analysis guidelines of NHANES, ${ }^{32}$ we used the Tayor series linearization method to take into account NHANES complex sampling design, including some variables missing, and calculate unbiased estimates of indicators such as standard errors and confidence intervals. Categorical variables are expressed as frequency (\%). Continuous variables are presented as mean \pm standard deviation (SD). Statistical differences between the means and proportions of the 2 groups were determined by the Mann-Whitney and chi-square tests. Logistic regression models were used to detect the independent association between dietary fiber intake and constipation. The multivariate logistic regression model was adjusted for gender, age, ethnicity, education, marital status, income-poverty ratio, BMI, smoking, poor oral health, vitamin $\mathrm{D}$ deficiency, depression, diabetes, chronic disease, milk, total fat, carbohydrate, protein, total saturated fatty acids, cholesterol, alcohol, moisture, and physical activity.

To maximize statistical power and minimize bias that might occur if some covariates with missing data were eliminated from data analyses, we used multiple imputations, based on 5 replications and a chained equation approach method utilizing a Mice software package, ${ }^{33}$ to account for missing data. The post-imputed datasets were combined with Rubin's rules. ${ }^{34}$ According to gender (male and female: 2 categories), age ( $<45, \geq 45,<65$, and $\geq 65: 3$ categories), education ( $<12$ th grade, high school, and college: 3 catego- ries), marital status (never married, married or living with a partner, and widowed/divorced/separated: 3 categories), income-poverty ratio ( $<2$ and $\geq 2: 2$ categories), BMI (normal, overweight, and obesity: 3 categories), smoking (no and yes: 2 categories), poor oral health (no and yes: 2 categories), vitamin D deficiency (no and yes: 2 categories), diabetes (no and yes: 2 categories), chronic diseases (none, 1 , and 2 or more: 3 categories), physical activity (non-activie and active: 2 categories), milk (never, rarely, sometimes, and often: 4 categories), depression (no and yes: 2 categories), alcohol $(<6$ and $\geq 6: 2$ categories), and other dietary variables (T1, T2, and T3: 3 categories), we imputed the missing data. Next, we compared the results of pre-imputation to the post-imputation to identify whether created complete data had a significant difference from pre-imputation data. Our results demonstrated that created complete data showed no significant difference from raw data (Tables 1 and 2). Since the main missing data was smoking, we did a sensitivity analysis to compare the results of participants with known smoking data to that of missing smoking data. Then, we used the participants with missing smoking data to analyze the association between dietary fiber and constipation in different physical activity groups. Our results demonstrated that participants with known smoking data showed no significant difference from missing smoking data (Supplementary Table 1).

Then, we applied interaction analyses to analyze the relationship between dietary fiber intake and constipation in different physical activity groups. We performed likelihood ratio tests comparing the modification and interaction of different stratified models. Stratified groups were divided by interaction terms. In individual stratified groups, multiple logistic regression was employed to explore associations between dietary fiber intake and constipation, adjusting for gender, age, ethnicity, education, marital status, income-poverty ra-

Table 1. Results of Multiple Regression Analyses of the Association Between Dietary Fiber Intake and Constipation Between Pre- and Postimputation Data

\begin{tabular}{|c|c|c|c|c|}
\hline \multirow{2}{*}{ Model I ${ }^{\mathrm{a}}$} & \multicolumn{2}{|c|}{ Pre-imputation } & \multicolumn{2}{|c|}{ Post-imputation } \\
\hline & OR $(95 \% \mathrm{CI})$ & $P$-value & OR $(95 \% \mathrm{CI})$ & $P$-value \\
\hline Dietary fiber ${ }^{\mathrm{b}}$ & $0.98(0.98,0.99)$ & $<0.001$ & $0.98(0.98,0.99)$ & $<0.001$ \\
\hline 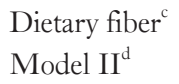 & $0.97(0.96,0.98)$ & $<0.001$ & $0.97(0.96,0.98)$ & $<0.001$ \\
\hline Dietary fiber ${ }^{b}$ & $0.98(0.96,1.00)$ & 0.130 & $0.99(0.98,1.00)$ & 0.065 \\
\hline Dietary fiber ${ }^{c}$ & $1.00(0.97,1.03)$ & 0.863 & $0.99(0.97,1.01)$ & 0.443 \\
\hline
\end{tabular}

\footnotetext{
${ }^{\mathrm{a}}$ Model I was adjusted for gender, age, ethnicity, marital status, education, income- poverty ratio, and body mass index.

${ }^{\mathrm{b}, \mathrm{c}}$ Constipation is defined by stool consistency and stool frequency respectively.

${ }^{\mathrm{d}}$ Model II was further adjusted for smoking, poor oral health, vitamin D deficiency, depression, diabetes, chronic diseases, milk, total fat, carbohydrate, protein, total saturated fatty acids, cholesterol, alcohol, moisture, and physical activity.

Post-imputations values were combined by Rubin's rules.
} 
Table 2. Subgroup Analyses of the Association Between Dietary Fiber Intake and Constipation Stratified by Physical Activity Between Pre- and Post-imputation Data

\begin{tabular}{|c|c|c|c|c|}
\hline \multirow{2}{*}{ Variable } & \multicolumn{2}{|c|}{ Pre-imputation } & \multicolumn{2}{|c|}{ Post-imputation } \\
\hline & OR $(95 \% \mathrm{CI})$ & $P$ for interaction & OR $(95 \% \mathrm{CI})$ & $P$ for interaction \\
\hline Physical activity $^{a}$ & & 0.044 & & 0.029 \\
\hline$<500$ & $1.02(0.98,1.05)$ & & $1.01(0.99,1.03)$ & \\
\hline$\geq 500$ & $0.97(0.94,0.99)$ & & $0.97(0.96,0.99)$ & \\
\hline Physical activity ${ }^{\mathrm{b}}$ & & 0.672 & & 0.639 \\
\hline$<500$ & $0.99(0.94,1.05)$ & & $0.98(0.95,1.02)$ & \\
\hline$\geq 500$ & $1.01(0.97,1.04)$ & & $0.99(0.97,1.01)$ & \\
\hline
\end{tabular}

${ }^{\mathrm{a}, \mathrm{b}}$ Constipation is defined by stool consistency and stool frequency respectively.

Adjusted for gender, age, ethnicity, education, marital status, income-poverty ratio, body mass index, smoking, poor oral health, vitamin D deficiency, depression, diabetes, chronic diseases, milk, total fat, carbohydrate, protein, total saturated fatty acids, cholesterol, alcohol, and moisture.

Post-imputations values were combined by Rubin's rules.

tio, BMI, smoking, poor oral health, vitamin D deficiency, depression, diabetes, chronic disease, milk, total fat, carbohydrate, protein, total saturated fatty acids, cholesterol, alcohol, moisture, and physical activity if not stratified.

A two-sided $P<0.05$ was considered statistically significant. Statistical analyses were performed using Empower Stats (http:// www.empowerstats.com) and R software, version 3.4.3 (http:// www.R-project.org/).

\section{Results}

\section{Clinical Characteristics}

Based on the stool consistency definition of constipation, 7.5\% (95\% CI, 7.1-7.9\%) of Americans had constipation. However, using the stool frequency definition of constipation resulted in a lower prevalence of constipation 3.5\% (95\% CI, 3.2-3.8\%). Table 3 presents the clinical characteristics of the study population based on the stool consistency definition. Gender, age, ethnicity, education, marital status, income-poverty ratio, BMI, poor oral health, depression, milk, total fat, carbohydrates, protein, total saturated fatty acids, cholesterol, alcohol, moisture, physical activity, and dietary fiber were significantly associated with constipation $(P<0.001)$, whereas smoking, vitamin $\mathrm{D}$ deficiency, diabetes, and chronic diseases were not. The use of the stool frequency definition of constipation resulted in few changes, ie, smoking and vitamin $\mathrm{D}$ deficiency were then significantly related to constipation $(P<0.001)$, as shown in Supplementary Table 2 .

\section{Univariate and Multivariate Regression Analysis of Dietary Fiber Intake for Constipation}

To study the association between dietary fiber intake and constipation, univariate and multiple logistic regression analyses were used. Based on the stool consistency definition, the intake of dietary fiber was associated with constipation (OR, 0.97; 95\% CI, 0.97-0.98; $P<$ 0.001) in the crude model (Table 4). Similar outcome was observed using the stool frequency definition (OR, $0.95 ; 95 \% \mathrm{CI}, 0.93-0.96$; $P<0.001)$. After adjusting for variables such as gender, age, ethnicity, education, marital status, income-poverty ratio, and BMI, dietary fiber intake was also related to constipation based on stool consistency (OR, 0.99 ; 95\% CI, 0.98-0.99; $P<0.001$ ) and stool frequency definition (OR, 0.97; 95\% CI, 0.96-0.98; $P<0.001)$. To exclude the influence of other variables, we further adjusted for related variables including smoking, poor oral health, vitamin D deficiency, depression, diabetes, chronic diseases, milk, total fat, carbohydrate, protein, total saturated fatty acids, cholesterol, alcohol, moisture, and physical activity. For constipation defined using stool consistency, the intake of dietary fiber was not significantly associated with constipation (OR, 0.98; 95\% CI, 0.96-1.00; $P=0.130)$. A similar outcome was observed using the stool frequency definition $(\mathrm{OR}, 1.00 ; 95 \% \mathrm{CI}$, 0.97-1.03; $P=0.863)$. Furthermore, we used multiple imputations to compare the regression analyses of pre-imputation to the postimputation to identify that the created complete data did not have a significant difference from pre-imputation data (Table 1). The intake of dietary fiber was not significantly correlated to constipation defined by stool consistency (OR, 0.99; 95\% CI, 0.98-1.00; $P=0.065)$ and stool frequency (OR, $0.99 ; 95 \% \mathrm{CI}, 0.97-1.01$; $P=0.443)$.

To avoid bias caused by the different definitions of constipation, 
Table 3. Clinical Characteristics of the Study Population From National Health and Nutrition Examination Survey 2005-2010 (Using the Stool Consistency Definition of Constipation)

\begin{tabular}{|c|c|c|c|}
\hline \multirow{2}{*}{ Characteristic } & No constipation & Constipation & \multirow{2}{*}{$P$-value } \\
\hline & $\mathrm{n}=12894^{\mathrm{a}}(\%)^{\mathrm{b}}$ & $\mathrm{n}=1047^{\mathrm{a}}(\%)^{\mathrm{b}}$ & \\
\hline Gender & & & $<0.001$ \\
\hline Male & $6710(50.5)$ & $345(28.8)$ & \\
\hline Female & $6184(49.5)$ & $702(71.2)$ & \\
\hline Age (yr) & & & 0.013 \\
\hline$<45$ & $5285(45.7)$ & $477(49.8)$ & \\
\hline$\geq 45,<65$ & $4433(37.0)$ & $324(32.7)$ & \\
\hline$\geq 65$ & $3176(17.3)$ & $246(17.5)$ & \\
\hline Ethnicity & & & $<0.001$ \\
\hline Non-Hispanic White & $6468(72.4)$ & $455(64.2)$ & \\
\hline Non-Hispanic Black & $2536(10.6)$ & $244(15.3)$ & \\
\hline Mexican American & $2308(7.8)$ & $190(9.7)$ & \\
\hline Other & $1582(9.2)$ & $158(10.8)$ & \\
\hline Education $^{c}$ & & & $<0.001$ \\
\hline$<12$ th grade & $3546(17.5)$ & $351(24.0)$ & \\
\hline High school & $3063(24.1)$ & $286(29.3)$ & \\
\hline College & $6276(58.4)$ & $407(46.7)$ & \\
\hline Marital status ${ }^{c}$ & & & 0.001 \\
\hline Never married & $2088(17.3)$ & $194(18.9)$ & \\
\hline Married & $7900(63.8)$ & $581(57.8)$ & \\
\hline $\begin{array}{l}\text { Widowed/divorced/ } \\
\text { separated }\end{array}$ & $2899(18.9)$ & $272(23.3)$ & \\
\hline Income-poverty ratio $(\%)^{c}$ & & & $<0.001$ \\
\hline$<2$ & $5394(32.1)$ & $517(42.9)$ & \\
\hline$\geq 2$ & $6571(67.9)$ & $451(57.1)$ & \\
\hline \multicolumn{4}{|l|}{ BMI $\left(\mathrm{kg} / \mathrm{m}^{2}\right)^{\mathrm{c}}$} \\
\hline Normal & $3585(30.8)$ & $365(38.4)$ & $<0.001$ \\
\hline Overweight & $4419(33.7)$ & $340(33.9)$ & \\
\hline Obesity & $4778(35.5)$ & $330(27.7)$ & \\
\hline Smoking $^{c}$ & & & 0.130 \\
\hline No & $3348(52.2)$ & $220(48.3)$ & \\
\hline Yes & $2905(47.8)$ & $208(51.7)$ & \\
\hline Poor oral health $^{c}$ & & & 0.003 \\
\hline No & $9399(89.5)$ & $720(83.9)$ & \\
\hline Yes & $1521(10.5)$ & $154(16.1)$ & \\
\hline Vitamin D deficiency $^{c}$ & & & 0.690 \\
\hline No & $7679(72.8)$ & $597(72.5)$ & \\
\hline Yes & $4176(27.2)$ & $334(27.5)$ & \\
\hline Depression & & & $<0.001$ \\
\hline No & $11778(92.5)$ & $903(86.5)$ & \\
\hline Yes & $1116(7.5)$ & $144(13.5)$ & \\
\hline Diabetes $^{c}$ & & & 0.896 \\
\hline No & $11350(92.0)$ & $922(91.3)$ & \\
\hline Yes & $1534(8.0)$ & $123(8.7)$ & \\
\hline
\end{tabular}

Table 3. Continued

\begin{tabular}{|c|c|c|c|}
\hline \multirow{2}{*}{ Characteristic } & No constipation & Constipation & \multirow{2}{*}{$P$-value } \\
\hline & $\mathrm{n}=12894^{\mathrm{a}}(\%)^{\mathrm{b}}$ & $\mathrm{n}=1047^{\mathrm{a}}(\%)^{\mathrm{b}}$ & \\
\hline Chronic diseases $^{c}$ & & & 0.822 \\
\hline None & $7263(60.2)$ & $585(57.4)$ & \\
\hline 1 & $3452(26.7)$ & $275(28.6)$ & \\
\hline 2 or more & $1974(13.1)$ & $170(14.0)$ & \\
\hline Milk $^{c}$ & & & 0.035 \\
\hline Never & $2075(15.3)$ & $178(17.2)$ & \\
\hline Rarely & $1893(14.2)$ & $146(13.9)$ & \\
\hline Sometimes & $3654(28.7)$ & $257(22.9)$ & \\
\hline Often & $5224(41.8)$ & $460(46.0)$ & \\
\hline Total fat & & & $<0.001$ \\
\hline $\mathrm{T} 1$ & $3649(25.7)$ & $412(36.8)$ & \\
\hline $\mathrm{T} 2$ & $4204(32.4)$ & $310(30.8)$ & \\
\hline $\mathrm{T} 3$ & $5041(41.9)$ & $325(32.4)$ & \\
\hline Carbohydrate & & & $<0.001$ \\
\hline $\mathrm{T} 1$ & $4018(29.5)$ & $388(35.1)$ & \\
\hline $\mathrm{T} 2$ & $4358(34.4)$ & $333(32.7)$ & \\
\hline $\mathrm{T} 3$ & $4518(36.1)$ & $326(32.2)$ & \\
\hline Protein & & & $<0.001$ \\
\hline $\mathrm{T} 1$ & $3230(22.4)$ & $358(31.8)$ & \\
\hline $\mathrm{T} 2$ & $4276(32.5)$ & $365(35.1)$ & \\
\hline $\mathrm{T} 3$ & $5388(45.1)$ & $324(33.1)$ & \\
\hline Total saturated fatty acids & & & $<0.001$ \\
\hline $\mathrm{T} 1$ & $4100(28.4)$ & $427(38.0)$ & \\
\hline $\mathrm{T} 2$ & $4071(31.1)$ & $317(30.8)$ & \\
\hline $\mathrm{T} 3$ & $4723(40.5)$ & $303(31.2)$ & \\
\hline Cholesterol & & & $<0.001$ \\
\hline $\mathrm{T} 1$ & $3362(25.7)$ & $350(33.3)$ & \\
\hline $\mathrm{T} 2$ & $4305(34.1)$ & $334(33.7)$ & \\
\hline T3 & $5227(40.2)$ & $363(33.0)$ & \\
\hline Alcohol & & & $<0.001$ \\
\hline$<6$ & $9809(74.2)$ & $886(84.4)$ & \\
\hline$\geq 6$ & 3085 (25.8) & $161(15.6)$ & \\
\hline Moisture & & & $<0.001$ \\
\hline $\mathrm{T} 1$ & $1916(11.0)$ & $240(20.1)$ & \\
\hline $\mathrm{T} 2$ & $4459(32.0)$ & $394(36.9)$ & \\
\hline $\mathrm{T} 3$ & $6519(57.0)$ & $413(43.0)$ & \\
\hline Dietary fiber & & & $<0.001$ \\
\hline $\mathrm{T} 1$ & $3234(23.1)$ & $359(34.9)$ & \\
\hline $\mathrm{T} 2$ & $4251(33.3)$ & 325 (29.6) & \\
\hline $\mathrm{T} 3$ & $5409(43.6)$ & $363(35.5)$ & \\
\hline Dietary fiber (g) & $16.5 \pm 10.1$ & $13.9 \pm 8.4$ & $<0.001$ \\
\hline Physical activity $^{c}$ & & & $<0.001$ \\
\hline Non-active & $2545(22.0)$ & 239 (28.4) & \\
\hline Active & $7763(78.0)$ & $544(71.6)$ & \\
\hline
\end{tabular}

an represents unweighted counts.

${ }^{\mathrm{b}}$ Percentages are weighted to the American population.

${ }^{\mathrm{c}}$ Numbers that do not add up to $100 \%$ are attributable to missing data.

Values are expressed as $\mathrm{n}(\%)$ or mean $\pm \mathrm{SD}$. 
Table 4. Multiple Logistic Regression of the Association Between Dietary Fiber Intake and Constipation

\begin{tabular}{|c|c|c|c|c|c|c|}
\hline \multirow{2}{*}{ Variable } & \multicolumn{2}{|c|}{ Crude model $^{a}$} & \multicolumn{2}{|c|}{ Model I } & \multicolumn{2}{|c|}{ Model II ${ }^{\mathrm{c}}$} \\
\hline & OR $(95 \% \mathrm{CI})$ & $P$-value & OR $(95 \% \mathrm{CI})$ & $P$-value & OR $(95 \% \mathrm{CI})$ & $P$-value \\
\hline Dietary fiber $^{\mathrm{d}}$ & $0.97(0.97,0.98)$ & $<0.001$ & $0.99(0.98,0.99)$ & $<0.001$ & $0.98(0.96,1.00)$ & 0.130 \\
\hline Dietary fiber ${ }^{\mathrm{e}}$ & $0.95(0.93,0.96)$ & $<0.001$ & $0.97(0.96,0.98)$ & $<0.001$ & $1.00(0.97,1.03)$ & 0.863 \\
\hline
\end{tabular}

${ }^{\mathrm{a}}$ This model was not adjusted for any confounding variables.

${ }^{b}$ Model I was adjusted for gender, age, ethnicity, marital status, education, income-poverty ratio, and body mass index.

'Model II was further adjusted for smoking, poor oral health, vitamin D deficiency, depression, diabetes, chronic diseases, milk, total fat, carbohydrate, protein, total saturated fatty acids, cholesterol, alcohol, moisture, and physical activity.

Constipation is defined by ${ }^{\mathrm{d}}$ stool consistency and ${ }^{\mathrm{e}}$ stool frequency, respectively.

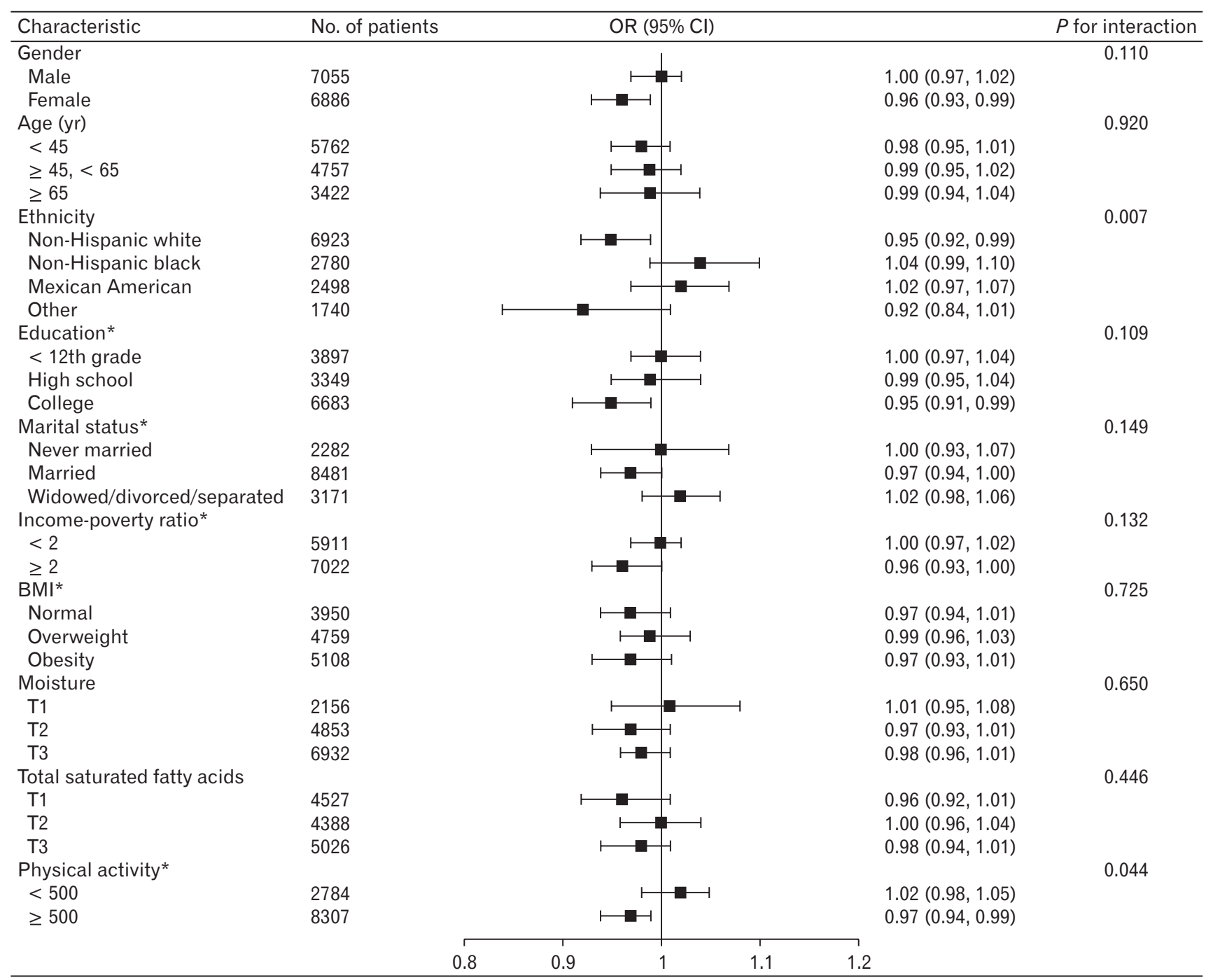

Figure 2. Subgroup analyses of the association between dietary fiber intake and constipation (stool consistency), according to clinical characteristics. Adjusted for gender, age, ethnicity, education, marital status, income-poverty ratio, body mass index (BMI), smoking, poor oral health, vitamin D deficiency, depression, diabetes, chronic diseases, milk, total fat, carbohydrate, protein, total saturated fatty acids, cholesterol, alcohol, moisture, and physical activity, except for the subgroup variable. It shows interaction analyses stratified by gender, age, ethnicity, education, marital status, income-poverty ratio, BMI, moisture, total saturated fatty acids, and physical activity. More subgroup analyses of other variables are shown in Supplementary Figure 1. *Numbers that do not add up to $100 \%$ are attributable to missing data. 
we carried out the sensitivity analysis focused on other 3 constipation-related symptoms. Whether in univariate or multiple logistic regression analysis, dietary fiber intake was not related to selfreported constipation (BHQ080), laxative use status (BHQ100), and the frequency of laxative use (BHQ110) (Supplementary Table 3).

\section{The Impact of Physical Activity on the Association Between Dietary Fiber Intake and Constipation}

According to different definitions of constipation, Figures 2 and
3 show the interaction effect between dietary fiber intake and constipation in different subgroups. Adjusting for gender, age, ethnicity, education, marital status, income-poverty ratio, BMI, smoking, poor oral health, vitamin $\mathrm{D}$ deficiency, depression, diabetes, chronic diseases, milk, total fat, carbohydrate, protein, total saturated fatty acids, cholesterol, alcohol, and moisture, physical activity significantly modified the association between dietary fiber intake and constipation using the stool consistency definition (Fig. 2). Among non-active participants, increasing the intake of dietary fiber was

\begin{tabular}{|c|c|c|c|c|}
\hline Characteristic & No. of patients & OR $(95 \% \mathrm{Cl})$ & & $P$ for interaction \\
\hline Gender & & & & 0.567 \\
\hline Male & 7055 & $\longmapsto-1$ & $0.98(0.94,1.03)$ & \\
\hline Female & 6886 & $\longmapsto 1$ & $1.00(0.96,1.04)$ & \\
\hline Age (yr) & & & & 0.090 \\
\hline$<45$ & 5762 & $\longmapsto+1$ & $0.95(0.90,1.01)$ & \\
\hline$\geq 45,<65$ & 4757 & $\mapsto$ & $1.02(0.98,1.06)$ & \\
\hline$\geq 65$ & 3422 & 5 & $1.02(0.93,1.11)$ & \\
\hline Ethnicity & & & & 0.984 \\
\hline Non-Hispanic white & 6923 & 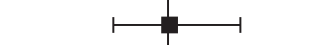 & $1.00(0.96,1.05)$ & \\
\hline Non-Hispanic black & 2780 & -1 & $0.99(0.93,1.05)$ & \\
\hline Mexican American & 2498 & & $1.00(0.84,1.19)$ & \\
\hline Other & 1740 & & * & \\
\hline Education** & & & & 0.014 \\
\hline$<12$ th grade & 3897 & $\longmapsto$ & $1.05(1.01,1.10)$ & \\
\hline High school & 3349 & -1 & $0.97(0.91,1.03)$ & \\
\hline College & 6683 & $\longmapsto$ & $0.96(0.91,1.02)$ & \\
\hline Marital status** & & & & 0.219 \\
\hline Never married & 2282 & $\square$ & $1.01(0.93,1.10)$ & \\
\hline Married & 8481 & $\longmapsto$ & $0.97(0.93,1.01)$ & \\
\hline Widowed/divorced/separated & 3171 & $\longrightarrow$ & $1.03(0.98,1.09)$ & \\
\hline Income-poverty ratio** & & & & 0.772 \\
\hline$<2$ & 5911 & $\longmapsto$ & $0.99(0.96,1.03)$ & \\
\hline$\geq 2$ & 7022 & -1 & $1.00(0.96,1.05)$ & \\
\hline $\mathrm{B} \overline{\mathrm{MI}} * *$ & & & & 0.067 \\
\hline Normal & 3950 & $\longmapsto-1$ & $0.95(0.90,1.01)$ & \\
\hline Overweight & 4759 & a & $1.02(0.97,1.08)$ & \\
\hline Obesity & 5108 & $\mapsto$ & $1.03(0.98,1.09)$ & \\
\hline Moisture & & & & 0.641 \\
\hline $\mathrm{T} 1$ & 2156 & - & $1.01(0.95,1.08)$ & \\
\hline $\mathrm{T} 2$ & 4853 & $\longmapsto$ & $0.97(0.94,1.01)$ & \\
\hline T3 & 6932 & $\mapsto-1$ & $0.98(0.96,1.01)$ & \\
\hline Total saturated fatty acids & & & & 0.190 \\
\hline $\mathrm{T} 1$ & 4527 & 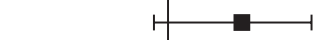 & $1.05(0.99,1.10)$ & \\
\hline $\mathrm{T} 2$ & 4388 & $\longmapsto+$ & $0.98(0.92,1.03)$ & \\
\hline T3 & 5026 & $\longmapsto$ & $0.99(0.95,1.05)$ & \\
\hline Physical activity** & & & & 0.672 \\
\hline$<500$ & 2784 & $\longmapsto$ & $0.99(0.94,1.05)$ & \\
\hline \multirow[t]{2}{*}{$\geq 500$} & 8307 & $\longmapsto$ & $1.01(0.97,1.04)$ & \\
\hline & 0.8 & 0.9 & 1.2 & \\
\hline
\end{tabular}

Figure 3. Subgroup analyses of the association between dietary fiber intake and constipation (stool frequency), according to clinical characteristics. Adjusted for gender, age, ethnicity, education, marital status, income-poverty ratio, body mass index (BMI), smoking, poor oral health, vitamin D deficiency, depression, diabetes, chronic diseases, milk, total fat, carbohydrate, protein, total saturated fatty acids, cholesterol, alcohol, moisture, and physical activity, except for the subgroup variable. It shows interaction analyses stratified by gender, age, ethnicity, education, marital status, incomepoverty ratio, BMI, moisture, total saturated fatty acids, and physical activity. More subgroup analyses of other variables are shown in Supplementary Figure 2. *The model failed because of the small sample size. ${ }^{*}$ Numbers that do not add up to $100 \%$ are attributable to missing data. 
not related to stool consistency-related constipation (OR, 1.02; 95\% CI, 0.98-1.05; $P=0.407)$. For physically active participants, however, an increase in dietary fiber intake was associated with stool consistency-related constipation (OR, 0.97; 95\% CI, 0.94-0.99; $P=0.020)$. Moreover, the relationship between dietary fiber intake and stool consistency-related constipation was significantly different for different physical activity groups $(P$ interaction $=0.044)$ (Fig. 2). For constipation defined using stool frequency, increasing dietary fiber intake was not related to constipation among the nonactive participants (OR, 0.99; 95\% CI, 0.94-1.05; $P=0.767$ ) nor physically active participants (OR, 1.01; 95\% CI, 0.97-1.04; P $=0.751$ ) (Fig. 3). Furthermore, the relationship between dietary fiber intake and constipation using the stool frequency definition was not significantly different for different physical activity groups $(P$ interaction $=0.672)$. Besides, we also use multiple imputations to evaluate that the modification effect of physical activity on the correlation between dietary fiber and constipation was consistent between pre- and post-imputation (Table 2). Increasing dietary fiber intake was associated with stool consistency-related constipation in the active participants (OR, 0.97; 95\% CI, 0.96-0.99), but not non-active participants (OR, 1.01; 95\% CI, 0.99-1.03). Additionally, the association between dietary fiber intake and constipation using the stool frequency definition was not significantly different among non-active participants (OR, 0.98; 95\% CI, 0.95-1.02) and active participants (OR, 0.99; 95\% CI, 0.97-1.01). Furthermore, we compared the modification effects of physical activity in the participants with missing smoking data was similar to that of participants with known smoking data (Supplementary Table 1). Other variables including gender, age, ethnicity, education, marital status, income-poverty ratio, BMI, smoking, poor oral health, vitamin D deficiency, depression, diabetes, chronic diseases, milk, total fat, carbohydrate, protein, total saturated fatty acids, cholesterol, alcohol, and moisture, did not significantly modify the association between dietary fiber intake and constipation based on definition of stool consistency and stool frequency (Supplementary Fig. 1 and 2, respectively).

Additionally, Supplementary Figure 3 shows the interaction effect results of the sensitivity analysis. For physically active participants, dietary fiber intake was not associated with self-reported constipation (OR, 1.01; 95\% CI, 0.99-1.02; P = 0.339). Among non-active participants, increasing the intake of dietary fiber was not related to self-reported constipation (OR, 1.00; 95\% CI, 0.98 1.03; $P=0.849$ ). Similarly, the correlation between dietary fiber intake and laxative use status was not significant among non-active participants (OR, 1.00; 95\% CI, 0.97-1.04; $P=0.952)$ nor physi- cally active participants $(\mathrm{OR}, 1.02 ; 95 \% \mathrm{CI}, 0.99-1.05 ; P=0.171)$. Moreover, dietary fiber intake was not related to the frequency of laxative use among the non-active participants (OR, 1.03; 95\% CI, $0.93-1.13 ; P=0.601)$ nor physically active participants (OR, 1.01; 95\% CI, 0.89-1.15; $P=0.855)$.

\section{Discussion}

In this study, we evaluated whether dietary fiber intake was related to constipation in adults and assessed the relationship between dietary fiber intake and constipation in groups with different physical activity levels. From a large, nationally representative sample of American adults, the prevalence of constipation defined by stool consistency was higher than that defined by stool frequency $(7.5 \%$ vs $3.5 \%$ ). After adjusting for a wide range of variables, multiple logistic regression revealed that dietary fiber intake was not associated with constipation using 2 different definitions. However, we found that the association between dietary fiber intake and constipation (based on stool consistency) was inconsistent at different levels of physical activity. Specifically, increasing dietary fiber intake was not associated with stool consistency-related constipation in the nonactive group, while it was related to stool consistency-related constipation in the physically active group. Nevertheless, we did not find that increasing dietary fiber intake was related to constipation (based on stool frequency) in either non-active or active groups.

The results of our study are significantly different from previous studies that used the NHANES database. Markland et $\mathrm{al}^{5}$ selected participants greater than 20 years of age from the 2005 2008 NHANES. According to their study, approximately $10.2 \%$ of females and $4.0 \%$ of males reported constipation. After adjusting for multiple variables, dietary fiber intake was not associated with constipation (based on stool consistency). In contrast, Shen et $\mathrm{al}^{14}$ conducted a cross-sectional study using NHANES data from 2005-2010 and suggested that lower dietary fiber intake was related to a higher risk of constipation (based on stool consistency). Additionally, Shen et $\mathrm{al}^{14}$ also suggested that participants with less than 500 MET-minutes per week of physical activity were not associated with a high incidence of constipation (based on stool consistency). Similarly, Wilson ${ }^{15}$ analyzed the NHANES data from 2007-2010 and reported that physical inactivity was not related to hard/lumpy stools or passing $<3$ stools per week. In a study of nurses' health, Dukas et $\mathrm{al}^{16}$ reported that increasing dietary fiber intake and moderate physical activity can decrease the prevalence of constipation. However, previous studies did not discuss the effect of physical activity on the interaction between dietary fiber intake and constipa- 
tion.

In a single-center, open-label trial, Zhang et $\mathrm{al}^{35}$ suggested that dietary fiber intake can alleviate constipation symptoms, mainly stool consistency. Our study provides a new perspective on the role of dietary fiber on constipation. The benefits of dietary fiber intake for alleviating stool consistency-related constipation may not occur in the whole population but may be relevant in the physically active population. In a meta-analysis of randomized controlled trials, Yang et $\mathrm{al}^{36}$ reported that increasing the intake of dietary fiber can increase stool frequency, but cannot improve stool consistency. Unfortunately, the effect of physical activity on the association between dietary fiber intake and constipation was not observed in our study when the stool frequency definition was used. Therefore, the influence of dietary fiber on constipation may only be observed in physically active participants when utilizing the stool consistency definition. Furthermore, the preventative effects of dietary fiber for constipation may occur by an improvement in stool consistency. Compared to the traditionally held belief that dietary fiber promotes stool frequency, our results indicate that the correlation between dietary fiber intake and stool frequency is not significant. Stool frequency may be more likely to correlate with other factors such as probiotics, ${ }^{37,38}$ polyethylene glycol, ${ }^{39}$ etc. To better understand the relationship between dietary fiber intake, stool consistency, and stool frequency, it is necessary to further explore their association from a clinical and experimental perspective.

To maximize statistical power and minimize bias that might occur if some covariates with missing data were eliminated from data analyses, we used multiple imputations, based on 5 replications and a chained equation approach method utilizing a Mice software package ${ }^{33}$ to account for missing data. The results between preand post-imputations were similar. Besides, we did a sensitivity analysis to compare whether the results of participants with known smoking data were different from that of participants with missing smoking data. Our results demonstrated that the results of participants with known smoking data were similar to that of missing smoking data. To avoid bias caused by the different definitions of constipation, we carried out the sensitivity analysis focused on other 3 constipation-related symptoms. Using the sensitivity analysis, we did not find that dietary fiber intake was associated with self-reported constipation, laxative use status, and the frequency of laxative use (Supplementary Table 3). Additionally, dietary fiber intake was not related to the above in different physical activity groups (Supplementary Fig. 3). The previous studies showed different views on the relationship between dietary fiber intake and laxative use. ${ }^{36,40} \mathrm{In}$ order to better investigate the association between dietary fiber and self-reported constipation, laxative use status, and the frequency of laxative use, we also need to research from the perspective of clinic and experiment and enlarge the sample size.

In addition, the dietary fiber used in this study is likely to be more prone to error, as it was obtained through a 24-hour recall. It is noteworthy that the potential exposure misclassification resulting from such errors would bias toward to the null and thus result in an underestimation of the association between dietary fiber intake and constipation (based on stool consistency or stool frequency).

The present study has several limitations. First, this was a crosssectional study of the NHANES data. This means that causal and temporal relationships between dietary fiber intake and constipation could not be determined. Therefore, we cannot say for certain that increasing dietary fiber intake will improve constipation. Second, according to the Rome criteria, the definition of constipation includes not only stool forms and frequency but also several other constipation-associated symptoms. Therefore, the current study may not represent the true frequency of constipation. Third, the data of BHQ080, BHQ100, and BHQ110 were only included from 2009-2010. Hence, to better understand the association between them and dietary fiber intake, we need to enlarge the sample size. Fourth, the NHANES survey was self-reported in the format of interviews and questionnaires, which can lead to inaccurate information and recall bias. For instance, reports of participants' dietary habits were obtained through a 24-hour follow-up. Therefore, we could not obtain long-term dietary information. Finally, because few participants in the NHANES database reported detailed dietary composition information, we were unable to incorporate the amount of supplemental fiber that participants may have been taking. These should be considered and included in future studies.

In conclusion, after adjusting for gender, age, ethnicity, education, marital status, income-poverty ratio, BMI, smoking, poor oral health, vitamin $\mathrm{D}$ deficiency, depression, diabetes, chronic diseases, milk, total fat, carbohydrates, protein, total saturated fatty acids, cholesterol, alcohol, moisture, and physical activity, multivariate logistic analysis results did not reveal a significant relationship between dietary fiber intake and constipation. However, for physically active participants, increasing dietary fiber intake was associated with stool consistency-related constipation while it was not strongly related to stool consistency-related constipation among non-active participants. In addition, increasing the intake of dietary fiber is not significantly associated with stool frequency in different physical activity groups. These results suggest that increasing dietary fiber intake may alleviate stool consistency-related constipation for physically active participants. 


\section{Supplementary Materials}

Note: To access the supplementary tables and figures mentioned in this article, visit the online version of Journal of Neurogastroenterology and Motility at http://www.jnmjournal.org/, and at https://doi.org/10.5056/jnm20051.

Financial support: This study was supported by the National Natural Science Foundation of China (Grant No. 81570483 and 81770541).

\section{Conflicts of interest: None.}

Author contributions: Wei-Dong Tong designed the research and had primary responsibility for the final content; Yi Li analyzed the data, performed all the statistical analysis and wrote the manuscript; and Yang Qian made all tables and figures. All authors reviewed the data, aided in interpretation of the results, reviewed the manuscript, and read and approved the final manuscript.

\section{References}

1. Drossman DA, Hasler WL. Rome IV-functional GI disorders: disorders of gut-brain interaction. Gastroenterology 2016;150:1257-1261.

2. Paquette IM, Varma M, Ternent C, et al. The American Society of Colon and Rectal Surgeons' clinical practice guideline for the evaluation and management of constipation. Dis Colon Rectum 2016;59:479-492.

3. Sperber AD, Bangdiwala SI, Drossman DA, et al. Worldwide prevalence and burden of functional gastrointestinal disorders, results of Rome foundation global study. Gastroenterology Published Online First:12 Apr 2020. doi: 10.1053/j.gasto.2020.04.014.

4. Suares NC, Ford AC. Prevalence of, and risk factors for, chronic idiopathic constipation in the community: systematic review and metaanalysis. Am J Gastroenterol 2011;106:1582-1591.

5. Markland AD, Palsson O, Goode PS, Burgio KL, Busby-Whitehead $\mathrm{J}$, Whitehead WE. Association of low dietary intake of fiber and liquids with constipation: evidence from the national health and nutrition examination survey. Am J Gastroenterol 2013;108:796-803.

6. Mugie SM, Benninga MA, Di Lorenzo C. Epidemiology of constipation in children and adults: a systematic review. Best Pract Res Clin Gastroenterol 2011;25:3-18.

7. Bharucha AE, Pemberton JH, Locke GR 3rd. American gastroenterological association technical review on constipation. Gastroenterology 2013;144:218-238.

8. Johanson JF, Kralstein J. Chronic constipation: a survey of the patient perspective. Aliment Pharmacol Ther 2007;25:599-608.

9. Irvine EJ, Ferrazzi S, Pare P, Thompson WG, Rance L. Health-related quality of life in functional GI disorders: focus on constipation and re- source utilization. Am J Gastroenterol 2002;97:1986-1993.

10. Rao SS, Seaton K, Miller MJ, et al. Psychological profiles and quality of life differ between patients with dyssynergia and those with slow transit constipation. J Psychosom Res 2007;63:441-449.

11. Rao SS. Constipation: evaluation and treatment of colonic and anorectal motility disorders. Gastroenterol Clin North Am 2007;36:687-711.

12. Rao SS, Rattanakovit K, Patcharatrakul T. Diagnosis and management of chronic constipation in adults. Nat Rev Gastroenterol Hepatol 2016;13:295-305.

13. Shah BJ, Rughwani N, Rose S. In the clinic. Constipation. Ann Intern Med 2015;162:ITC1

14. Shen L, Huang C, Lu X, Xu X, Jiang Z, Zhu C. Lower dietary fibre intake, but not total water consumption, is associated with constipation: a population-based analysis. J Hum Nutr Diet 2019;32:422-431.

15. Wilson PB. Associations between physical activity and constipation in adult Americans: results from the national health and nutrition examination survey. Neurogastroenterol Motil 2020:e13789.

16. Dukas L, Willett WC, Giovannucci EL. Association between physical activity, fiber intake, and other lifestyle variables and constipation in a study of women. Am J Gastroenterol 2003;98:1790-1796.

17. Lewis SJ, Heaton KW. Stool form scale as a useful guide to intestinal transit time. Scand J Gastroenterol 1997;32:920-924.

18. Whitehead WE, Borrud L, Goode PS, et al. Fecal incontinence in US adults: epidemiology and risk factors. Gastroenterology 2009;137:512517, 517, e1-e2.

19. Bujanda L. The effects of alcohol consumption upon the gastrointestinal tract. Am J Gastroenterol 2000;95:3374-3382.

20. Sommers T, Mitsuhashi S, Singh P, et al. Prevalence of chronic constipation and chronic diarrhea in diabetic individuals in the United States. Am J Gastroenterol 2019;114:135-142

21. Jamshed N, Lee ZE, Olden KW. Diagnostic approach to chronic constipation in adults. Am Fam Physician 2011;84:299-306.

22. U.S. department of health and human services physical activity guidelines for Americans. Available from URL: http://www.hhs.gov/fitness/beactive/physical-activity-guidelines-for-americans/index (accessed 22 Dec 2020)

23. Ballou S, Katon J, Singh P, et al. Chronic diarrhea and constipation are more common in depressed individuals. Clin Gastroenterol Hepatol 2019;17:2696-2703.

24. Kroenke K, Spitzer RL, Williams JB. The PHQ-9: validity of a brief depression severity measure. J Gen Intern Med 2001;16:606-613.

25. Ikebe K. Significance of oral function for dietary intakes in old people. J Nutr Sci Vitaminol (Tokyo) 2015;61(suppl):S74-S75.

26. Lundström O, Manjer J, Ohlsson B. Smoking is associated with several functional gastrointestinal symptoms. Scand J Gastroenterol 2016;51:914-922.

27. National health and nutrition examination survey. MEC in-person dietary interviewers procedure manual. Available from URL: https:/www. cdc.gov/nchs/data/nhanes/nhanes_03_04/dietary_mec.pdf (accessed 22 Dec 2020).

28. Panarese A, Pesce F, Porcelli P, et al. Chronic functional constipation is strongly linked to vitamin D deficiency. World J Gastroenterol 
2019;25:1729-1740.

29. Centers for disease control and prevention. Analytical note for 25-hydroxyvitamin D data analysis using NHANES III(1988-1994), NHANES 2001-2006 and NHANES 2007-2010(October 2015). Available from URL: https://wwwn.cdc.gov/nchs/nhanes/vitamind/analyticalnote.aspx?h=/Nchs/Nhanes (accessed 22 Dec 2020).

30. Forrest KY, Stuhldreher WL. Prevalence and correlates of vitamin D deficiency in US adults. Nutr Res 2011;31:48-54.

31. National center for health statistics. Module 3: weighting. Available from URL: https:/wwwn.cdc.gov/nchs/nhanes/tutorials/module3.aspx (accessed 22 Dec 2020).

32. National center for health statistics. Module 4: variance Estimation. Available from URL:https://www.cdc.gov/nchs/nhanes/tutorials/module4.aspx (accessed 31 Mar, 2021).

33. Bernhardt PW. Model validation and influence diagnostics for regression models with missing covariates. Stat Med 2018;37:1325-1342.

34. Rubin DB. Multiple Imputation for Nonresponse in Surveys. Hoboken: John Wiley and Sons, Inc: 1987.
35. Zhang X, Tian H, Gu L, et al. Long-term follow-up of the effects of fecal microbiota transplantation in combination with soluble dietary fiber as a therapeutic regimen in slow transit constipation. Sci China Life Sci 2018;61:779-786.

36. Yang J, Wang HP, Zhou L, Xu CF. Effect of dietary fiber on constipation: a meta-analysis. World J Gastroenterol 2012;18:7378-7383.

37. Ford AC, Quigley EM, Lacy BE, et al. Efficacy of prebiotics, probiotics, and synbiotics in irritable bowel syndrome and chronic idiopathic constipation: systematic review and meta-analysis. Am J Gastroenterol 2014;109:1547-1561.

38. Choi CH, Chang SK. Alteration of gut microbiota and efficacy of probiotics in functional constipation. J Neurogastroenterol Motil 2015;21:4-7.

39. Lee-Robichaud H, Thomas K, Morgan J, Nelson RL. Lactulose versus polyethylene glycol for chronic constipation. Cochrane Database Syst Rev 2010:CD007570.

40. Sturtzel B, Elmadfa I. Intervention with dietary fiber to treat constipation and reduce laxative use in residents of nursing homes. Ann Nutr Metab 2008;52(suppl 1):54-56. 